\title{
Whole Systems Within Whole Systems: The Oregon Health Plan's Expansion of Services for Back and Neck Pain
}

\author{
Emery R. Eaves, PhD, Clarissa W. Hsu, PhD, ${ }^{2}$ Lynn L. DeBar, PhD, MPH, ${ }^{2}$ \\ Catherine J. Livingston, MD, MPH, ${ }^{3}$ Laura E. Ocker, LAc, MAcOM, ${ }^{4}$ Sarah J. McDonald, BA, ${ }^{2}$ \\ Laurel Dillon-Sumner, $\mathrm{MA}, \mathrm{MPH}^{2}$ and Cheryl Ritenbaugh, $\mathrm{PhD}, \mathrm{MPH}^{5}$
}

\begin{abstract}
Objectives: The authors employ a Whole Systems framework to explore implementation of new guidelines for back and neck pain in Oregon's Medicaid system. Whole Systems research is useful for understanding the relationship between complementary and integrative health care (CIH) and conventional health care systems in real-world clinical and practice settings.

Design: Preliminary results are from an observational study designed to evaluate state-wide implementation of $\mathrm{CIH}$ and other non-pharmacological treatments for neck and back pain among Oregon Medicaid patients. This natural experiment, even in early stages, provides insight into the challenges of integrating Whole Systems oriented therapies into Medicaid billing and treatment.

Methods: Qualitative data are drawn from: (1) semi-structured interviews with representatives of each of the 16 coordinated care organizations (CCOs) responsible for administering the Oregon's Medicaid insurance through the Oregon Health Plan (OHP); and (2) open-ended survey responses from acupuncturists in all 16 CCO areas.

Results: Implementation of the new policy guidelines poses logistical and epistemological challenges. Differences in worldview, inadequate reimbursement, and simple lack of awareness of CIH among medical providers are some of the factors that pose barriers to merging CIH therapies into conventional frameworks.

Conclusions: In this article, we explore the potential for a Whole Systems perspective to better explain the complexity of integrating $\mathrm{CIH}$ and other non-pharmacological services into a state financed health care system. Oregon's expansion of services for back and neck pain presents an opportunity to explore challenges and successes in melding multiple approaches to health and pain management into a managed system such as the OHP.
\end{abstract}

Keywords: whole systems research, complementary and integrative health care, chronic pain, opioid response, complementary and alternative medicine

\section{Introduction}

$\mathbf{T}$

He United States is EXPERIEnCING a widely acknowledged crisis stemming from the broad use of opioids for chronic pain. ${ }^{1-5}$ From a Whole Systems perspective, evidencebased, non-pharmacological management for chronic pain is central to addressing systemic causes of opioid overuse. Emergent national clinical research funders such as the PatientCentered Outcomes Research Institute (PCORI) prioritize patient choice, values, and health care access. These shifts underscore the potential value of and demand for many complementary integrated health modalities. ${ }^{6}$ In keeping with this, Oregon's

\footnotetext{
${ }^{1}$ Department of Anthropology, Northern Arizona University, Flagstaff, AZ.

${ }^{2}$ Kaiser Permanente Washington Health Research Institute, Seattle, WA.

${ }^{3}$ Department of Family Medicine, Oregon Health \& Science University, Portland, OR.

${ }^{4}$ Multnomah County Health Department, Portland, OR.

${ }^{5}$ Department of Family and Community Medicine, University of Arizona, Tucson, AZ.
} 
Table 1. Oregon Health Plan New Back and Neck Care Guidelines (Effective July 1, 2016)

Increased coverage (through Guideline Note $56^{\mathrm{a}}$ )
Pain oriented cognitive behavior therapy
Spinal manipulation
Acupuncture
Physical therapy/occupational therapy
Non-opioid medications
Yoga
Interdisciplinary Rehab
Supervised exercise
Massage therapy

Decreased Coverage

Opioids (through Guideline Note $60^{\mathrm{b}}$ )

Limitations on acute and subacute prescribing

Noncoverage of chronic opioids, with 1 year taper Epidural steroid injections

Surgeries

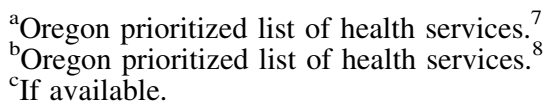

Medicaid system, administered through the Oregon Health Plan (OHP) recently implemented innovative new guidelines, described below, for treating back and neck pain. The goal was to improve access to safer, non-pharmacological treatments that have demonstrated scientific evidence of improving functioning ${ }^{6,9-18}$ while limiting chronic opioid therapy and other interventional approaches for chronic pain (see Table 1).

All 50 U.S. states have prescription drug monitoring plans, although most do not require reporting. Every state Medicaid plan provides access to Buprenorphine treatment, and all have laws mandating access to Naloxone. ${ }^{19} \mathrm{Al}-$ though most Medicaid agencies offer some coverage for pain management alternatives to opioid therapy, fewer than half have implemented policies that encourage or require their use. ${ }^{20}$ Most Medicaid programs focus on identifying high-risk patients and prescribers, while few emphasize comprehensive strategies for chronic pain treatment or integration of nonpharmacological treatments. ${ }^{21}$ In a 2016 National Academy for State Health Policy survey, ${ }^{20} 12$ states reported that they had implemented policies specifically intended to encourage the use of non-pharmacological alternatives to opioid therapy for chronic pain. In a recent cross-sectional study of Medicaid, commercial, and Medicare plans, Heyward et al. ${ }^{22}$ reported that most plans covered chiropractic, physical therapy, and occupational therapy for chronic pain, but few provided acupuncture or other non-pharmacological interventions. ${ }^{22}$

Among efforts reported in the 2016 National Academy for State Health Policy survey, Florida, Vermont, and Rhode Island have piloted programs specifically targeting cost savings, opioid reduction, and pain management through allowing reimbursement for complementary and integrative health care $(\mathrm{CIH})$ services such as acupuncture, chiropractic, and massage. ${ }^{20}$ While similar in some ways to these earlier efforts, Oregon's approach was more ambitious in scope and in its focus on encouraging adoption of recent primary care practice guideline recommendations to improve the safety of opioid prescribing to treat chronic pain. ${ }^{23}$ Florida's initiative was piloted in the early 2000's and then discontinued at a very different time in the trajectory and national response to opioid prescribing concerns; while Rhode Island's efforts have been more restricted in scope, targeting those with frequent emergency room visits rather than all Medicaid insured patients in the State with pertinent pain-related diagnoses. Finally, Vermont's efforts have been limited to expanding coverage for acupuncture-related services rather than the broader array of $\mathrm{CIH}$ services as well as behavioral and physical therapy related services included in the Oregon initiative.

The OHP, Oregon's Medicaid program, put in place two guidelines (guideline notes 56 and 60, see Table 1) specifically aimed to: reduce new opioid starts (guideline note 60); taper long-term opioid users (guideline note 60); and promote the use of non-pharmacological, evidence-based therapies for chronic neck and back pain (guideline note 56). In Oregon, coordinated care organizations (CCOs), similar to Accountable Care Organizations, provide Medicaidrelated services. CCOs have the flexibility to organize care within their respective network of providers and payers in a manner designed to best suit the needs of their particular community. The policy for expanded services, when CCOs were expected to make those therapies billable, went into place July 1, 2016. Decreased coverage for opioid therapy was not expected to be fully implemented until 2 years later, allowing time to taper patients off opioids. The OHP's new guidelines expanded coverage of $\mathrm{CIH}$ therapies (e.g., acupuncture, chiropractic care, yoga, and massage) and other non-pharmacological therapies (e.g., behavioral health and physical therapy) for back and neck pain (Table 1). A critical aspect of Oregon's guidelines is that they were not intended as a cost-saving measure, but rather as a measure to more effectively manage chronic pain among Medicaid recipients.

Whole Systems research ${ }^{24,25}$ is based on the premise that real-world aspects of $\mathrm{CIH}$ treatment such as patient/practitioner interactions, elements of the health care environment, and other outcomes of direct importance to patients and other non-research stakeholders should be included in the evaluation of initiatives such as those detailed above. In 2003, Ritenbaugh et al. ${ }^{24}$ described the outcomes of a conference of researchers that characterized Whole Systems research as, "the intention to include conceptually as part of the investigative context all aspects of any internally consistent approach to treatment, including its philosophical basis, patients, practitioners, setting of practice, and methods/ materials used. Whole systems research acknowledges unique patient, family, community, and environmental characteristics and perspectives." In 2005, Verhoef et al. ${ }^{25}$ argued that most research on complementary and alternative medicine (CAM, now commonly referred to as $\mathrm{CIH}$ ) focused on the effectiveness and efficacy of individual modalities rather than on the whole systems of care in which they are practiced. ${ }^{25}$ A Whole Systems framework consists of patient-centered approaches to healing that are attentive 


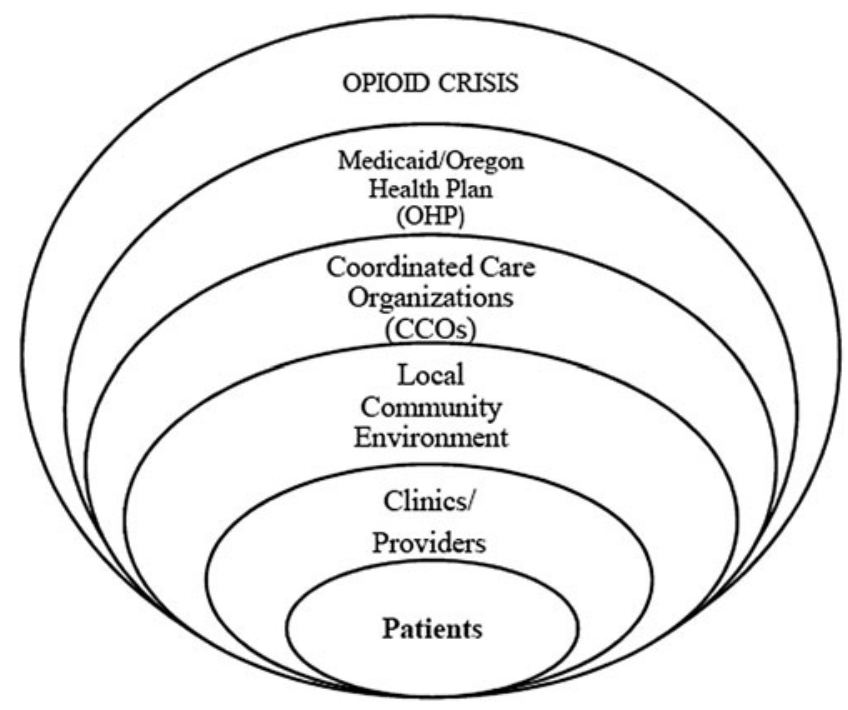

FIG. 1. OHP back and neck pain guideline implementation as conceptualized before qualitative evaluation. OHP, Oregon Health Plan.

to practitioners' skills and practice in real-world contexts. ${ }^{25,26}$ Whole Systems researchers advocate for the use of systems theory to consider the complexity of real-world provision of therapies that cannot be reduced to "pill form.",27

To explore the real-world provision of services in Oregon under the OHP's new guidelines, we used an a priori multilevel framework ${ }^{28}$ designed to consider the multiple actors and levels of influence that OHP administrators and our research team expected would influence implementation of the new guidelines before beginning evaluation (Fig. 1). As illustrated in Figure 1, we anticipated that various levels of influence would impact care pathways and patient-related outcomes.

Oregon's expansion of services for back and neck pain is, thus, an opportunity to explore challenges and successes in melding multiple approaches to health and pain management into the managed system of the OHP. In this paper, we explore the potential for a Whole Systems perspective to better explain the complexity of integrating $\mathrm{CIH}$ and other non-pharmacological services into a state financed health care system.

\section{Materials and Methods}

\section{Data sources}

Insights in this paper are based on initial observations of the rollout of the new guidelines, from two qualitative data sources collected in spring 2018: (1) telephone interviews with representatives from CCOs, health plans and state agencies $(n=30)$; and (2) open-ended survey responses collected from acupuncturists by a member of the project's stakeholder advisory committee $(n=84)$. All qualitative data collection was deemed exempt by the Kaiser Permanente Washington Institutional Review Board.

\section{Interviews with CCO/Health Plan representatives}

Representatives from each of the 16 CCOs and the state fee-for-service department were invited to participate in 1-h telephone interviews. Respondents were encouraged to invite other members of their organizations who could contribute key information regarding the implementation of the guidelines to participate in the interview. Semi-structured qualitative interviews, collected from February to May 2018, focused on: (1) what each organization had done to implement the new guidelines; (2) specific techniques used to communicate changes to clinics, providers, and patients; (3) data collection and metrics; (4) challenges; (5) contextual influences; (6) unintended consequences; and (7) future plans. Interviews were audio-recorded and transcribed verbatim. Coding in ATLASti was conducted by the first author, a medical anthropologist experienced in analysis of qualitative data related to $\mathrm{CIH}$ therapies and chronic pain. ${ }^{29-32}$ Analysis was based on a framework approach, ${ }^{33}$ using apriori and emergent codes related to initial themes noted by the research team and guided by a multilevel framework, as initially conceptualized in Figure 1. A framework approach is well-suited to consider specific questions and a priori issues, ${ }^{33}$ such as implementation challenges and successes. Using this framework as a guide to explore complexity within the Oregon health care system, codes were designed to capture interactions and experiences at each of the many levels in our initial model. Analysis was thus attentive to experiences and interactions among payers, community environments, clinics, providers, and patients within the larger policy context of mandates focused on reducing opioid use in the United States.

At least one representative from each of the 16 CCOs in Oregon and the state fee-for-service department participated in the semi-structured qualitative interviews. In total, 18 interviews were completed with 30 respondents (16 chief medical officers; 4 clinical pharmacists; 3 pharmacy directors; 3 quality improvement staff members; 2 outreach and referral coordinators; and 2 staff members in charge of data analytics).

\section{Acupuncture survey}

To understand how at least one type of $\mathrm{CIH}$ provider interacted with the OHP during the initial phase of the rollout (since the July 1, 2016 Medicaid payment coverage of such services in Oregon), an acupuncturist member of the project's stakeholder advisory committee (L.E.O.) surveyed acupuncturists practicing in Oregon. The survey, available to practitioners as an online form between February and March 2018, aimed to assess the context and implications of the OHP guidelines on acupuncturists' practice patterns and involvement with patients whose treatment services were covered through the OHP. There have been many changes over the years in coverage of acupuncture-related treatment under OHP, such that acupuncturists are particularly attentive to Medicaid-related policy changes and, hence, ideal initial $\mathrm{CIH}$ practitioner survey respondents.

Open-ended questions asked acupuncturist respondents about: reasons for accepting or not accepting OHP; things that have gone particularly well or particularly poorly for them in working with OHP; barriers or challenges in working with particular CCOs or the OHP more generally; ease of credentialing and billing for services provided to OHP patients; adequacy of reimbursement for services provided; perspectives on referral and prior authorization 
requirements and procedures; ability to create an effective treatment plan within OHP's covered services; ability to provide appropriate care for OHP patients given administrative structure of the program; perceptions of OHP program changes over time; and a further open-ended section for additional comments.

The survey was distributed to Oregon acupuncturists known to the study researchers, and then distributed more widely via the Oregon College of Oriental Medicine and the Oregon Association of Acupuncture and Oriental Medicine social media outlets. Snowball sampling through networks, as well as solicitations to complete the survey sent individually to acupuncturists living in areas that were underrepresented among early respondents, were undertaken to achieve a wide range of respondents.

Eighty-four licensed acupuncturists practicing in Oregon completed the survey. Acupuncturist respondents included at least one respondent who saw patients from each of the 16 CCOs in the state thereby ensuring that responses represented variations in practice experiences across the state. Sixty-five percent of respondents reported that they accept OHP patients in their practice.

\section{Results}

CCO stakeholders and acupuncturist survey respondents identified challenges arising from the complexity of working with the new guidelines which we distilled into two overarching themes: logistical challenges (billing, credentialing, and providing access) and epistemological challenges (differences in $\mathrm{CIH}$ and biomedical approaches to healing).

\section{Logistical challenges}

Adding coverage of $\mathrm{CIH}$ for back and neck pain necessitates many layers of stakeholder involvement, communication, and administrative changes at multiple levels and in multiple systems. Logistical challenges include CCO credentialing and billing requirements, Medicaid credentialing and billing structures, referral processes, and authorization and guideline tracking. $\mathrm{CCO}$ representatives explained that working within this complex system makes it difficult to pay $\mathrm{CIH}$ providers. Credentialing is the process by which individual health care providers are established as legally able to provide services and be reimbursed within the state's reimbursement systems. A frequently noted issue was that massage therapists and yoga practitioners are not eligible to be credentialed through Medicaid in Oregon and therefore could not directly bill Medicaid for their services even though those services were theoretically covered by the health plan. CCO representatives therefore noted encouraging massage therapists, exercise therapists, or yoga practitioners to affiliate themselves with credentialed providers, such as physical therapists, to bill for their services.

\footnotetext{
We have a number of massage therapists in private solo practice that said hey, we want to sign up with you and provide massage therapy. And we said that's great, we really want to pay you, but the guideline says we can't pay massage therapists as a provider type. We tried to say, how about you make some kind of working relationship with a PT office or orthopedic office? Ultimately none of those ever quite came to pass. (CCO Interview 08)
}

There's a part of the guideline note that talks about supervised exercise and yoga as well. Both of those were very problematic, because while they could potentially be coded, they can't be credentialed, so we had a heck of a time trying to figure out how to cover those things. On the yoga side, we did get one or two providers that actually hired a yoga provider to come into their office. That was the only way we could figure out how to do it. (CCO Interview 01)

To provide patients with covered access to $\mathrm{CIH}$ services for back and neck pain, some CCO representatives described creative strategies to reach providers who were out of network or had been ineligible for reimbursement (as described above for massage therapists and yoga practitioners). One CCO representative articulated how important these alternative health care services are and the efforts they had made to ensure their patients had access to such services.

We hit upon a challenge that our acupuncture providers in the community here were like, "we're full, we can't accept any new patients." So then we start approving people to go out of network for the alternative treatments like acupuncture or massage, because this community has a limited number of providers ... If we're stopping access to opiates, we have to give them some other alternative so they can feel they're getting treatment. ... I think we have to give them some path to healing. (CCO Interview 07)

For licensed $\mathrm{CIH}$ and other providers able to be credentialed independently (e.g., licensed acupuncturists, chiropractic physicians, physical therapists, and behavioral health providers), the complexity of Medicaid billing requirements still presented a hurdle to providing care within the new guidelines. Acupuncturists surveyed, for example, described frustration with getting credentialed, determining what services are covered for which conditions, and how to bill for services covered through different plans (Table 2).

\section{Epistemological challenges}

Although the $\mathrm{CCO}$ representatives and primary care providers (PCPs) understood that the guidelines were based on evidence, many were not familiar with the evidence or with what patients should expect from these broader $\mathrm{CIH}$ treatments. Knowledge gaps can hinder the referral process, potentially preventing patients from accessing the full array of options intended by the guidelines. The CCO representative quoted below, for example, was skeptical about services they were not confident would work for patients.

I think those are excellent therapies, the active ones. I'm not such a fan of massage, but I think yoga, supervised exercise, is a brilliant thing to include, and I think more guidance would be helpful on where that's evidence-based and where that's not. Right? Because I don't want to authorize things and use CCO funds and patients' time and energy on something that doesn't work. (CCO Interview 02)

OHP's implementation of new back and neck pain guidelines was hindered by issues common in U.S. health care. As one CCO representative explained, whole patient care is inherently challenging in this complex system.

It's really hard in medicine anyway when you don't see the person as a whole, but here's a person at their chiropractor or their acupuncturist and then they're at their PCP. We're not looking at how we have benefited the person on the whole. I don't think there's good communication between 


\section{Table 2. Reasons for Accepting or Not Accepting Oregon Health Plan Patients} (ACUPUnCturist Perspectives)

Yes: $n=55 ; 24$ of 55 respondents who said they accept OHP patients cited wanting to serve low income communities or people who would not otherwise have access to acupuncture as their primary reason for doing so. Others cited wanting to build their practice.

No: $n=29$; reasons for not taking OHP patients included low reimbursement rates and overall difficulty of getting paid or likelihood of patient to no-show for appointments.

Credentialing and getting established as payable providers also posed barriers at various steps in the process that dissuaded some from accepting OHP patients.
Until I reach beyond my capacity for patient numbers, I'll take anyone's insurance. Also, they need acupuncture just like anybody else and are among the least likely to get it any other way.

I did [get credentialed with $\mathrm{OHP}$ ] to be able to serve the underserved. I find sometimes poverty equals more stress and illness.

I have accepted OHP in the past. However, the process was confusing, and it took months to even find out what we were doing wrong in billing. The process to even get approved for 1 patient was much more tedious and intensive than any other insurance we take. We finally got paid 5 months after we saw this client, and we were paid $\$ 10$ less per visit than the lowest paying insurance we take. If more than $20 \%$ of my clientele were OHP I would go out of business in a matter of months.

We accepted OHP in the past, but now it doesn't pay very well and people with OHP tend to want to come in for "extra" treatments all the time and are much more likely to no-show or late-cancel because they don't have to pay the associated fees.

Everything is pretty opaque, including trying to find benefits, getting credentialed, finding out what the plan will pay for, and even just figuring out which plan each patient is on.

The credentialing was fine. Establishing ourselves as a payable entity was more murky. Getting it cleared up with every entity pointing the finger at someone else was terrible.
OHP, Oregon Health Plan.

acupuncturists and PCP's about what's needed to make a person better. (CCO Interview 04)

Despite 20 years of Whole Systems research showing the complexity of $\mathrm{CIH}$ practice and the likelihood that single modalities used alone have lower effectiveness than when utilized as part of an integrated system, ${ }^{24,25,27,34-36}$ most evidence and therefore the new guidelines focused on discrete single components of these Whole Systems-oriented therapies. Acupuncturists expressed both frustration with the

Table 3. Acupuncturists Perspectives on Providing Whole Systems Care Within AN EVIDENCE-BASED FRAMEWORK

Most acupuncturists said they provide the Whole Systems care they are trained to provide, while billing only for covered services (needles).

Some expressed frustration that OHP does not provide coverage for additional services they felt would help patients.

Acupuncturists also expressed satisfaction at being considered an important piece of improving pain management in a biomedically oriented system.
I do a LOT of bodywork and moxa which help the patient's condition significantly, especially when the conditions are complicated like [with] cancer, autoimmune disease, Parkinson's, etc. Yet, none of such services are recognized as billable and worth any payment. It makes me feel like I should just put some needles in and leave the room, instead of tending the patients for bodywork that actually helps even more than needling oftentimes.

[OHP] does not pay for herbs or moxa but then again, most insurance companies won't. However this clientele isn't able to pay for these additional things themselves. So I either gift them the herbs they need or they go without and aren't as helped as they could be.

OHP essentially promotes acupuncture technicians. The art of the Traditional Chinese Medicine doctor is not really valued. I constantly try to remind everyone we are treating the whole, not just pain management. In the end this is important for the true integrity of the profession in my opinion. (Acupuncturist 5)

I am so grateful that OHP has started paying for acupuncture. This group of people are terribly under served by the doctors in our area, and they need alternatives to opiates. I'm also pleased, thrilled, and tickled to death that the war on opioids is forcing docs to refer patients, which brings our abilities and successes into their view. 
guidelines' restrictions on providing Whole Systemsoriented care for patients and excitement that their successes were gaining some level of attention for having potential to address this issue (Table 3).

Quotes in Table 3 and the broader data collected from acupuncturists suggest that despite billing only for the evidence-based parts of their practice that are covered for OHP patients (needling), practitioners continued to believe in the importance of their Whole Systems practice for fully treating patients and often continued to provide such care despite the lack of payment due to the narrower coverage allowed by OHP guidelines. This tension illustrates the necessity of including providers,' payers' and other stakeholders' perspectives in evaluation of initiatives, such as the OHP's new guidelines, that attempt to integrate $\mathrm{CIH}$ and other non-pharmacological services into biomedically structured systems.

\section{Complexity of multiple actors and levels of influence in OHP system}

Based on the qualitative evaluation detailed above, realworld implementation of the new guidelines is more complex than the spheres of influence in our initial model (Fig. 1) illustrate. Figure 2 thus shows the complexity, beyond our initial model, with which actors interact within and outside of the context of the health plan, influenced by local environments, and as a consequence of encountering state policy changes largely aimed at addressing the broader context of a national opioid crisis. Moving beyond spheres of influence, a Whole Systems framework takes into account the spaces where $\mathrm{CIH}$ service provision remains separate, as well as the complexity of patients having the option, at least in theory, to seek $\mathrm{CIH}$ and other pain-related treatment without involving the larger biomedical systems (e.g., self-

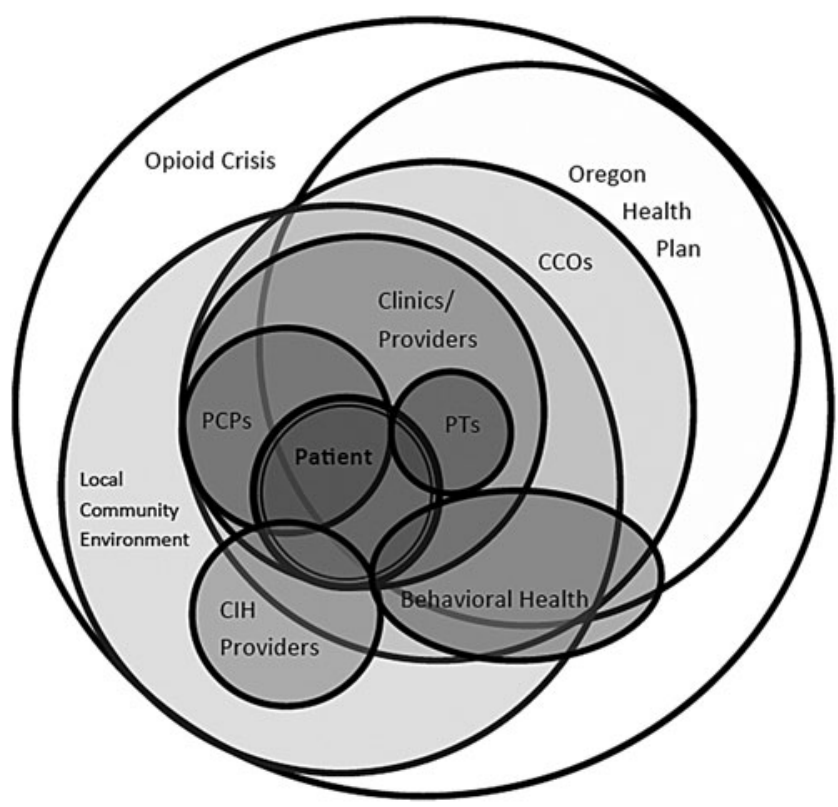

FIG. 2. OHP back and neck pain guideline implementation in real-world health care system as conceptualized using a Whole Systems framework. CCOs, coordinated care organizations; OHP, Oregon Health Plan. pay for opioids, or seeking care from a community-based acupuncture clinic with a sliding fee scale rather than care from an OHP authorized provider). As depicted in Figure 2, the local community environment and the OHP interact and influence one another, but one does not fall entirely within the influence of the other. To understand patients' experiences of health care and providers' experiences of health care provision within such a system, a Whole Systems perspective is well suited to consider not only levels of influence, but competing influences and areas where billing codes or electronic health records would provide only partial understanding.

\section{Discussion}

From a Whole Systems perspective, the OHP's new guidelines represent a step toward more sustainable management for chronic back and neck pain and an alternative to long-term opioid therapy. CIH treatments have traditionally been delivered separately from health plans ${ }^{36}$ and $\mathrm{CIH}$ and conventional practitioners do not have a history of working as colleagues. ${ }^{37}$ Thus, merging CIH therapies into standards of care for back and neck pain is logistically and epistemologically challenging at institutional, financial, and individual levels. ${ }^{6}$

Differences in worldviews, inadequate reimbursement, primacy of biomedical conventions in reimbursing for procedurally based care, and simple lack of awareness and understanding of $\mathrm{CIH}$ among medical providers, present challenges to merging $\mathrm{CIH}$ therapies into conventional care processes and payment structures. ${ }^{38-40}$ For example, some aspects of $\mathrm{CIH}$ practice, such as needling or spinal manipulation, are now covered for treatment of back and neck pain. CIH practitioners, however, may struggle to restrict care to use of a single modality (needling for acupuncturists; spinal manipulation for chiropractors) as well as to separate back and neck pain from other aspects of the person's health which they have been trained to consider part of "whole person" treatment and which they feel obligated to address.

Whole Systems research is particularly pertinent to fully characterize and understand this phenomenon, as acupuncturists report providing their whole range of services despite coverage only for evidence-based aspects. This discrepancy raises questions about how evaluation of evidence-based treatment delivery in the real-world can consider the actual provision of services in context. As demonstrated in Figure 2 above, real-world spheres of influence are not discrete or linear. A Whole Systems framework moves analysis beyond spheres of influence to consider a dynamic range of complex, overlapping, non-linear influences both within and outside of the OHP system. Whole Systems research aims to consider these less tangible aspects, as well as interactions between larger complex systems, to avoid separating aspects such as needling in acupuncture practice from other, less measurable aspects of the experience of seeking and providing care for chronic pain.

Lessons from this natural experiment may interest other state Medicaid plans or health care systems in their attempts to integrate $\mathrm{CIH}$ services for back and neck pain into standards of care, and demonstrate one possibility for working toward safer and more patient-centered care in the context of safety concerns stemming from the recent unilateral use of prescription 
opioids to treat chronic pain. ${ }^{4,41}$ To this end, we have shown how Whole Systems is useful to consider the complexity of implementing evidence-based aspects of $\mathrm{CIH}$ and other services into real-world coverage for Medicaid patients.

Our findings reflect the insights of health care administrators and clinical providers at the early stages of responding to the new guidelines. Later phases of our research will provide a more generalizable sense of the implementation of $\mathrm{CIH}$ and expanded services for back and neck pain in Oregon. Another limitation is that the snowball sampling technique used to distribute the acupuncturist survey means that we are unable to define a clear denominator and therefore a response rate. Despite these limitations, it is clear there are challenges to integrating $\mathrm{CIH}$ and other non-pharmacologic services into a Medicaid reimbursement structure. Other health systems working to encourage availability and provision of non-pharmacological (non-opioid) options for the treatment of chronic pain that include $\mathrm{CIH}$ services may benefit from understanding the potential challenges and complexities that arise in attempting to encourage uptake of such services through alterations in insurable treatment coverage such as those being undertaken in Oregon.

\section{Conclusions}

The OHP took an unprecedented step when it made a number of $\mathrm{CIH}$ and other evidenced-based non-pharmacological treatments available for back and neck pain to address the need for opioid alternatives. Recognition of the complexity of bringing together pre-existing complex $\mathrm{CIH}$ and conventional health care systems, and examples of how to address the inherent challenges of such an ambitious undertaking, may aid other locales in addressing the complex problems of pain management and responding to the current opioid crisis.

\section{Acknowledgment}

This work was supported through a PCORI Project Program Award (UOP-1609-36568).

\section{Disclaimer}

All statements in this report, including its findings and conclusions, are solely those of the authors and do not necessarily represent the views of the PCORI, its Board of Governors or Methodology Committee.

\section{Author Disclosure Statement}

No competing financial interests exist.

\section{References}

1. Volkow ND, Collins FS. The role of science in addressing the opioid crisis. N Engl J Med 2017;377:391-394.

2. Ryan SA. Calculating the real costs of the opioid crisis. Pediatrics 2018;141:e20174129.

3. Brown RE, Jr., Sloan PA. The opioid crisis in the United States: Chronic pain physicians are the answer, not the cause. Anesth Analg 2017;125:1432-1434.

4. Rudd RA, Aleshire N, Zibbell JE, Gladden RM. Increases in drug and opioid overdose deaths-United States, 20002014. Am J Transplant 2016;16:1323-1327.
5. Samet JH, Kertesz SG. Suggested paths to fixing the opioid crisis: Directions and misdirections. JAMA Netw Open 2018; 1:e180218.

6. Tick H, Nielsen A, Pelletier KR, et al. Evidence-based nonpharmacologic strategies for comprehensive pain care: The Consortium Pain Task Force white paper. Explore (NY) 2018;14:177-211.

7. Oregon Health Authority. Guideline Note 56. Online document available at: www.oregon.gov/oha/HPA/DSIHERC/Pages/Searchable-List.aspx?wp7687=se:\%22NONINTERVENTIONAL+TREATMENTS\%22, accessed October 23, 2018.

8. Oregon Health Authority. Guideline Note 60. Online document available at: www.oregon.gov/oha/HPA/DSI-HERC/ Pages/Searchable-List.aspx?wp7687=se:\%22OPIOIDS+FOR+ CONDITIONS+OF+THE+BACK+AND+SPINE\% 22, accessed October 23, 2018.

9. Brunner E, De Herdt A, Minguet P, et al. Can cognitive behavioural therapy based strategies be integrated into physiotherapy for the prevention of chronic low back pain? A systematic review. Disabil Rehabil 2013;35:1-10.

10. Lamb S, Lall R, Hansen Z, et al. A multicentred randomised controlled trial of a primary care-based cognitive behavioural programme for low back pain. The Back Skills Training (BeST) trial. Health Technol Assess 2010;14:1253.

11. Dworkin R, Turk D, Wyrwich K, et al. Interpreting the clinical importance of treatment outcomes in chronic pain clinical trials: IMMPACT recommendation. J Pain 2008;9: $105-121$.

12. Turk DC, Okifuji A. Treatment of chronic pain patients: Clinical outcomes, cost-effectiveness, and cost-benefits of multidisciplinary pain centers. Crit Rev Phys Rehabil Med 1998;10:181-208.

13. Hayden J, Van Tulder MW, Malmivaara A, Koes BW. Exercise therapy for treatment of non-specific low back pain. Cochrane Database Syst Rev 2005;3:1465-1858.

14. Lam M, Galvin R, Curry P. Effectiveness of acupuncture for nonspecific chronic low back pain: A systematic review and meta-analysis. Spine 2013;38:2124-2138.

15. Menke JM. Do manual therapies help low back pain? A comparative effectiveness meta-analysis. Spine 2014;39: E463-E472.

16. Walker BF, French SD, Grant W, Green S. Combined chiropractic interventions for low-back pain. Cochrane Database Syst Rev 2010;4:CD005427.

17. Holtzman S, Beggs RT. Yoga for chronic low back pain: A meta-analysis of randomized controlled trials. Pain Res Manag 2013;18:267-272.

18. Brosseau L, Wells GA, Poitras S, et al. Ottawa Panel evidencebased clinical practice guidelines on therapeutic massage for low back pain. J Bodyw Mov Ther 2012;16:424-455.

19. Parker AM, Strunk D, Fiellin DA. State responses to the opioid crisis. J Law Med Ethics 2018;46:367-381.

20. Dorr H, Townley C. Chronic pain management therapies in medicaid: policy considerations for non-pharmacological alternatives to opioids. A publication of the National Academy for State Health Policy. 2016. Online document at: https:// nashp.org/wp-content/uploads/2016/09/Pain-Brief.pdf, accessed October 23, 2018.

21. Lin DH, Jones CM, Compton WM, et al. Prescription drug coverage for treatment of low back pain among US Medicaid, Medicare advantage, and commercial insurers. JAMA Netw Open 2018;1:e180235. 
22. Heyward J, Jones CM, Compton WM, et al. Coverage of nonpharmacologic treatments for low back pain among US public and private insurers. JAMA Netw Open 2018;1: e183044.

23. Dowell D, Haegerich TM, Chou R. CDC guideline for prescribing opioids for chronic pain-United States, 2016. JAMA 2016;315:1624-1645.

24. Ritenbaugh C, Verhoef M, Fleishman S, et al. Whole systems research: A discipline for studying complementary and alternative medicine. Altern Ther Health Med 2003;9: 32-36.

25. Verhoef MJ, Lewith G, Ritenbaugh C, et al. Complementary and alternative medicine whole systems research: Beyond identification of inadequacies of the RCT. Complement Ther Med 2005;13:206-212.

26. Boon H, Verhoef M, O'Hara D, et al. Integrative healthcare: Arriving at a working definition. Altern Ther Health Med 2004;10:48.

27. Bell IR, Koithan M. Models for the study of whole systems. Integr Cancer Ther 2006;5:293-307.

28. Clauser SB, Taplin SH, Foster MK, et al. Multilevel intervention research: Lessons learned and pathways forward. J Natl Cancer Inst Monogr 2012;2012:127-133.

29. Eaves ER, Ritenbaugh C, Nichter M, et al. Modes of hoping: Understanding hope and expectation in the context of a clinical trial of complementary and alternative medicine for chronic pain. Explore (NY) 2014;10: 225-232.

30. Schafer LM, Hsu C, Eaves ER, et al. Complementary and alternative medicine (CAM) providers' views of chronic low back pain patients' expectations of CAM therapies: A qualitative study. BMC Complement Altern Med 2012;12:234.

31. Sherman KJ, Cherkin DC, Ichikawa L, et al. Treatment expectations and preferences as predictors of outcome of acupuncture for chronic back pain. Spine 2010;35:1471-1477.

32. Eaves ER, Sherman KJ, Ritenbaugh C, et al. A qualitative study of changes in expectations over time among patients with chronic low back pain seeking four CAM therapies. BMC Complement Altern Med 2015;15:12.
33. Srivastava A, Thompson SB. Framework analysis: A qualitative methodology for applied policy research. JOAAG 2009;4:72-79.

34. Elder C, Aickin M, Bell IR, et al. Methodological challenges in whole systems research. J Altern Complement Med 2006;12:843-850.

35. Ritenbaugh C, Hammerschlag R, Calabrese C, et al. A pilot whole systems clinical trial of traditional Chinese medicine and naturopathic medicine for the treatment of temporomandibular disorders. J Altern Complement Med 2008;14:475-487.

36. Floden L, Howerter A, Matthews E, et al. Considerations for practice-based research: A cross-sectional survey of chiropractic, acupuncture and massage practices. BMC Complement Altern Med 2015;15:140.

37. Kligler B, Brooks AJ, Maizes V, et al. Interprofessional competencies in integrative primary healthcare. Glob Adv Health Med 2015;4:33-39.

38. Hollenberg D. How do private CAM therapies affect integrative health care settings in a publicly funded health care system? J Complement Integr Med 2007;4. DOI: 10.2202/ 1553-3840-1056.

39. Sewell M. Challenges of integrating CAM and biomedicine. J Altern Complement Med 2007;13:307-308.

40. Song M-Y, John M, Dobs AS. Clinicians' attitudes and usage of complementary and alternative integrative medicine: A survey at the Johns Hopkins Medical Institute. J Altern Complement Med 2007;13:305-306.

41. Bohnert AS, Valenstein M, Bair MJ, et al. Association between opioid prescribing patterns and opioid overdoserelated deaths. JAMA 2011;305:1315-1321.

Address correspondence to: Emery R. Eaves, PhD Department of Anthropology Northern Arizona University PO Box 15200 Flagstaff, AZ 86011

E-mail: emery.eaves@nau.edu 